\title{
Organ-Associated Muscles in Aedes albopictus (Diptera: Culicidae) Respond Differentially to Sindbis Virus
}

\author{
MAI VO ${ }^{1}$, PAUL J. LINSER ${ }^{2}$, and DORIA F. BOWERS ${ }^{3}$ \\ ${ }^{1}$ Florida State University, College of Medicine, Tallahassee, FL 32306 \\ 2 Whitney Laboratory, University of Florida, St. Augustine, FL 32080
}

\section{Abstract}

Differential host cell responses to the alphavirus Sindbis were observed in visceral muscles of the adult female mosquito Aedes albopictus. Following intrathoracic inoculation with SIN, muscles associated with the midgut, hindgut, and ovary resulted in clearance, persistence, and refractoriness to virus, respectively. Prominent sarcomeres characteristic of myofilaments were identified in muscles associated with these three organs by phalloidin labeling of actin, confirming these cells as muscle. The location of virus antigen mimicked the distribution of actin in both mid- and hindgutassociated muscles. Furthermore, these myofilaments remained intact following virus clearance from midgut muscles and during virus persistence in hindgut muscles. Changes in the temporal onset of virus antigen following high titer inoculum compared with standard titer inoculum was observed in anterior midgut muscles, but not in muscles associated with the posterior midgut or hindgut. Muscle bundles closely approximated the gut surface, while a wispy association was displayed at the ovary surface. Prominent ultrastructural differences were observed in the basal lamina attached to the gut compared with the ovary. Additionally, ultrastructural evidence for virus-associated pathology was observed in gut-associated muscles and gut epithelium. Visceral muscles, all composed of the same tissue type, but associated to three different organs in the insect abdomen, responded differentially to Sindbis. We speculate that variations in structure, function or physiology and ultrastructure inherent to insect host cells or organs interactions reflect the complicated milieu of the organism and contribute to differential virus phenotypic expression in muscle cells.

\section{Keywords}

Sindbis virus; mosquito; visceral muscle; actin

Infection of mosquitoes with arthropod-borne viruses (arboviruses) results in a chronic infection that persists for the lifespan of the insect (Murphy et al. 1975, Shope 1985). Replication of virus progeny in permissive host cells is but one link in the chain of virus transmission in nature (Vo and Bowers, 2005). Sindbis virus (SIN; family Togaviridae; genus alphavirus) infection was observed in mosquito intestinal cells and salivary glands (Gaidamovich et al. 1973, Raikova et al. 1973) and more extensively in a relatively pantropic distribution in Aedine mosquitoes (Jackson et al. 1993, Bowers et al. 1995). The replication of the alphavirus SIN has been well documented in Aedes albopictus cell culture (Brown and Condreay 1986). Furthermore, while Culex tarsalis is considered the principle vector of many alphaviruses in North America (Fulhorst, et al. 1994), 26 different mosquito species including Ae. normanensis (Taylor) are principle vectors of SIN in Australia (Dhileepan et al. 1996) expanding the epidemiological significance beyond principle vector interests. Bowers and 
colleagues (1995) documented tissue-specific temporal and spatial differences in the onset, duration and clearance of SIN in adult female Ae. albopictus (Skuse) over an 18-d time-course. Following intrathorasic inoculation of 2550 plaque forming units (PFU)/insect (standard dose), virus antigen was first identified in fat bodies/hemolymph, salivary glands, head ganglion, gutassociated muscles, and tracheoles between 1- 4 d postinfection (p.i.). Sindbis virus antigen was cleared from the head ganglion, and midgut-associated muscles, and persisted in fat bodies, tracheoles, and hindgut-associated muscles. Ovarioles and malphigian tubules remained refractory to infection during the acute and persistent phases of infection. Co-existence of both permissive and refractory tissues were found in the adult mosquito, and tissue or organ responses to virus infection varied. Colocalization of SIN antigen and gross pathology in the lateral lobes of the salivary glands was documented at the light microscopic-level and SINassociated pathology was observed in gut-associated muscles at the ultra-structural-level (Bowers et al. 2003). Organ-specific differences in infection thresholds, tissue-specific differences in receptor molecules and host cell modulation of the spatial and temporal infection process were suggested.

The "suck-and-spit" behavior inherent to bloodfeeding is directly essential for insect survival and reproduction, while indirectly essential to arbovirus transmission in nature (Clements 1996, Higgs and Beaty 1996, Chapman 1999). These physiological events are integrated by the contractile activity of organ-associated muscles that govern the intake and digestion of foodstuffs during periodic peristalsis and oviduct contractions during oviposition (Messer and Brown 1995). Muscle tissues provide the forceful dynamics required for coherent movement essential to life. All insect visceral muscles are striated (Smith et al. 1966) revealing a sarcomere length 3-4 times larger than that observed in insect flight muscles or vertebrate skeletal muscles (Park and Shahabuddin 2000). A double investment of incomplete muscle bundles surrounds the mosquito gut proper (Smith et al. 1966) and similarities and differences in visceral muscle distribution are seen in various regions of the gut and between different species of mosquitoes (Park and Shahabuddin 2000). While circular muscle bundles are juxtaposed to the basal aspect of the gut epithelia, longitudinal muscle bundles are arranged upon the outer plane; differences in thickness and density of both muscle layers are observed between species. Similar layering of visceral muscle bundles are observed attached to the oviduct, while a thin wispy network of muscles surround the ovarian sheath (Gighoh 1964).

Presently, we describe variations in host cell response to SIN infection observed in organassociated muscles of adult female mosquitoes. We pose the question, "can various muscleorgan interactions affect the virus lifecycle"? After infection with SIN, responses in visceral muscles associated with different regions of the gut compared with visceral muscle cells juxtaposed to the ovary were observed as well as mosquito response to higher titer SIN challenge. We suggest that the virus phenotypic expression may be differential in spatially diverse visceral muscles, reflecting the varying milieu found in the host organism.

\section{Materials and Methods}

\section{Mosquitoes, Cell Culture, and Virus}

Aedes albopictus (Skuse) eggs and mosquitoes were maintained under insectary conditions $\left(25.5^{\circ} \mathrm{C} \pm 1.0,70-80 \% \mathrm{RH}\right.$, a 16L:8D photoperiod) and fed ad lib on honey-soaked cellucotton. After hatching, mosquito larvae were reared to adults, as previously described by Bowers and colleagues (1995) and female mosquitoes 5-7 d postemergence were used in all experiments. Colonized adult female mosquitoes were inoculated via the intrathoracic route (Rosen and Gubler 1974) with SIN (mean $=2,550 \mathrm{PFU} /$ per insect [standard-titer inoculum], $850,000 \mathrm{PFU} /$ insect [high-titer inoculum], or equivalent volume of $3 \%$ fetal calf serum in phosphate buffered saline (PBS; mock-infected), incubated under insectary conditions, and sampled at days $1,2,3,5,7,10$, and 18 p.i. for analysis. 
Cell cultures of baby hamster kidney (BHK-21/13/F) grown in Eagle's minimal essential medium supplemented with $10 \%$ fetal calf serum, $2 \mathrm{mM}$ glutamine, and $10 \%$ tryptose phosphate broth (Rentz and Brown 1976) were used to grow virus. The heat-resistant strain of SIN (SVHR), supplied by Elmer Pfefferkorn (Dartmouth Medical College, Hanover, NH; Burge and Pfefferkorn 1966a,b) was used as the wild-type virus (Bowers et al. 1995, 2003).

\section{Localization of Virus Antigens by Indirect Immunofluorescence}

Insect tissues and organs were dissected on days 1, 2, 3, 5, 10, and 18 p.i. and whole-mount tissues were labeled using a polyclonal antiserum specific to SIN (rabbit anti SIN, produced in our lab) to localize virus antigens. Staining and scoring was conducted as previously described (Bowers et al. 1995). Briefly, all tissues were scored at the microscope using an arbitrary scale of FITC-fluorescence $(0,+,++,+++$ or iv) designed to assess the intensity and distribution of the fluorescence signal specific to each organ. Distribution entailed the patterning of fluorescence seen in muscles such as $0=$ no signal,,$+++=$ individual foci or small not connected bands and +++ , iv $=$ completed circular or longitudinal bands all scored on a continuum. The onset of virus antigen detection in response to the high-titer inoculum was compared with the onset of detection following standard-titer inoculum (Bowers et al. 1995). Epifluorescent microscopy (Olympus System Microscope model BX60) was used to observe the fluorescent signal. Images were captured using a SPOT RT model 7.2.1 Color Mosaic Digital Camera and analyzed with SPOT Software (Version 3.5.7, Diagnostics Instruments, Sterling Heights, MI).

\section{Localization of Actin by Phalloidin Assay}

Insect midguts, hindguts, and ovaries were sampled at days 5, 10, and 18 p.i. for analysis of actin. Whole-mount preparations were held for one hour in a 1:1 fixative solution (Seron et al. 2004; $4 \%$ paraformaldehyde in $0.1 \%$ sodium cacodylate buffer, $\mathrm{pH} 7.3$ ) and insect hemolymph (42.3 mM NaCl, $3.0 \mathrm{mM} \mathrm{KCl}, 0.6 \mathrm{mM} \mathrm{MgSO}_{4}, 5.0 \mathrm{mM} \mathrm{CaCl}_{2}, 5.0 \mathrm{mM} \mathrm{NaHCO}_{3}, 5.0 \mathrm{mM}$ succinic acid, 5.0 mM malic acid, 5.0 mM L-proline, $9.1 \mathrm{mM} \mathrm{L}$-glutamine, $8.7 \mathrm{mML}$-histidine, $3.3 \mathrm{mM}$ L-arginine, $10 \mathrm{mM}$ dextrose, $25 \mathrm{mM}$ HEPES with $\mathrm{NaOH} \mathrm{pH} \mathrm{7.0).} \mathrm{Next,} \mathrm{tissues} \mathrm{were}$ submerged in a blocking preincubation medium (2\% goat serum and $0.1 \%$ Tween 20 in PBS, $\mathrm{pH} 7.5$ ) for one hour at room temperature. Tissues were washed three times with PBS (30 min each wash) between all steps. Tissues were incubated in 1:100 dilutions of FITC-phalloidin conjugate in preincubation medium overnight at $4^{\circ} \mathrm{C}$ followed by two PBS washes for $30 \mathrm{~min}$ each. Images were observed and captured using epifluorescent microscopy as indicated above.

\section{Ultrastructural Analysis of Virus Infection}

Tissues were prepared for transmission electron microscopy (TEM) as previously described (Bowers et al. 1995). Guts and ovaries were dissected from mosquitoes for analysis of muscleorgan interaction. Myofibrils, cell junctions, and basal lamina (BL) were observed, while BL thickness was recorded directly from micrograph negatives using a measuring magnifier.

\section{Results}

\section{Localization of SIN Antigens Mimicked the Distribution of Actin in Gut-Associated Muscles}

Actin molecules were labeled to determine the distribution of muscles in insect tissues. Myofilaments were observed in both circular and longitudinal muscle bundles associated with the gut exterior surface (Fig. 1A, D, and G). Cross-sharing of muscles (Park and Shahabuddin 2000) also termed cruciform regions (Bernick et al. 2007) were apparent in the midgut (Fig. $1 \mathrm{~A}$ and D) but absent in the hindgut (Fig. 1G). Longitudinal muscle bundles appeared more prominent in the midgut, while circular muscles were more apparent in the hindgut. Circular and longitudinal gut-associated muscles are organized into bundles that displayed a grate-work 
arrangement with respect to the gut longitudinal axis, ovary-associated muscles displayed a scattered, wispy arrangement (Fig. 1J). Sarcomeres were discernable in the anterior midgut and hindgut-associated muscles as well as the ovary-associated muscles. Sarcomeres were not clearly visible in muscles associated with the posterior midgut most likely because of the accordion-like structure of this stomach region. Actin labeling mimicked the distribution of SIN antigen in visceral muscles (Fig. 1) confirming these cells to be muscle. Furthermore, SIN antigen was cleared from anterior and posterior midgut muscle bundles (Fig. 1B, C, E, and F) persisted in hindgut muscles (Fig. 1H and I) and was not observed in ovary muscles (Fig. 1K and $\mathrm{L}$ ) when comparing day $2-4$ p.i. with day 10 p.i.

\section{Actin Distribution in Gut Muscles Was Unaltered During SIN Persistence}

Actin molecules were labeled to determine if SIN infection altered muscle integrity. Because Bowers' and colleagues (2003) detected SIN-associated gut muscle pathology at the ultrastructural-level at day 5 p.i., we questioned the survival of gut muscles following virus clearance. Comparison of actin localization in gut muscles between mock-infected and SINinfected revealed no apparent changes in distribution of this structural molecule at day 18 p.i. (Fig. 2) by light-microscopic analysis. Whole mount preparations of longitudinal and circular muscles bundles may be presented at different angles because of dissections, but remained intact and sarcomeres apparent following $18 \mathrm{~d}$ p.i. Gut-associated visceral muscles remained intact following clearance of virus antigen from the midgut and during persistence in the hindgut at day 18 p.i.

\section{High Titer Inoculum Resulted in an Earlier Onset of SIN Antigen in Some Insect Tissues}

A high titer inoculum dose of $850,000 \mathrm{PFU} /$ insect $(\approx 333 \mathrm{X}$ standard dose) resulted in an earlier detection of SIN antigen in the fat bodies/hemolymph, salivary glands, anterior midgutassociated muscles, and thoracic muscles (Fig. 3) compared with that seen following the standard delivery dose; $2550 \mathrm{PFU} /$ insect (Bowers et al. 1995). Onset of detection of SIN antigen in hind-gut/posterior midgut-associated muscles as well as the head ganglion was not altered. However, onset of detectable SIN antigen in the fat bodies/hemolymph, salivary glands, anterior midgut, and thoracic muscles appeared earlier, while ovarioles and malphigian tubules remained refractory following high titer virus challenge.

\section{Structural and Ultrastructural Differences in Muscle: Organ Association}

Comparisons of muscles organ interactions were assessed at the ultrastructural-level. Muscle bundles closely approximated the basal surface of the gut and displayed a loose, wispy association to the ovary surface. The muscle BL, a complex trilaminar extracellular matrix construct, was thinner compared with the BL surrounding the gut (1:2.8) and the ovary (1:3.2). The gut BL appeared multilayered (1- 4 layers), detached from the gut surface and presented characteristic vertical hatch marks. The ovary BL appeared single-layered with occasional rifts, closely juxtaposed to the organ surface and homogenous in its fine structure. Cell-to-cell junctions were observed between adjacent muscle cells and between muscle cells and gut epithelium but were not identified between muscle cells and the ovary surface (Figs. 4 and 5).

\section{Ultrastructural Evidence for Virus-Associated Pathology in Gut Cells and Gut-Associated Muscles}

SIN-associated pathology in gut muscles was previously identified (Bowers et al. 2003) and this study further investigated this observation. Virus-associated pathology and virus particles were observed in gut-associated muscles and gut epithelium following $5 \mathrm{~d}$ p.i. by TEM (Fig. $6)$. 


\section{Discussion}

SIN antigens have been detected in the circular and longitudinal muscles surrounding the gut of adult female Ae. albopictus following intrathoracic inoculation of virus (Bowers et al. 1995). Because of this tissue tropism, we chose to confirm that these cells were muscle cells and determine if they survived the infection. Adult female Ae. albopictus mosquitoes were inoculated with SIN, and we investigated three organs essential to virus transmission; midgut, hindgut, and ovaries. Can we detect differences in the in vivo visceral muscle response to SIN during acute (day $1-5$ p.i.) and/or persistent (day $\geq 10$ p.i.) infection following different multiplicities of infection (MOI)? While parenteral inoculation is not a natural route of infection it is an important model used to avoid interference of dissemination by potential gut barriers (Kramer et al. 1981). This route of infection also bypasses virus amplification by the gut epithelium, delivers an equivalent inoculation MOI to all structures bathed by hemolymph and permits assessment of infection potential at the tissue or organ level. Insects were challenged with a high titer inoculum dose to determine if this increased MOI would alter the temporal onset of infection as well as elicit infection in refractory muscles.

Circular and longitudinal muscle bundles invest the mosquito gut in a grate-like manner (Figs. 1 and 2), lending physical and physiological support to the gut simple epithelium. Branching, interlinking, and cross-sharing of muscle bundles are more apparent in the anterior midgut, while muscles in the posterior midgut are less precise, reflecting the extensive accordion-style gut expansion in response to receiving a blood bolus. Random cross-sharing of circular muscles in Ae. albopictus mimicked that observed in Ae. aegypti (Park and Shahabuddin 2000).

Furthermore, Bernick and colleagues (2007) speculate that such cruciform cells that share muscle fibers between adjacent bands, act to coordinate contractile activities of peristalsis. We describe a "herring-bone" pattern that characterized the circular muscles predominate in the hindgut; structurally quite different from the gratework arrangement of midgut muscles. Even more diverse yet, a wispy sleeve-like arrangement of muscles surrounded the ovaries. While all visceral muscles are the same tissue-type, their morphological presentation differed at these three locations.

The pattern of SIN infection mimicked the distribution of actin in gut-associated muscles (Fig. 2) confirming a muscle tissue tropism. Prominent sarcomeres were observed during acute infection, following clearance in the midgut muscles, as well as during persistence in the hindgut muscles. Sarcomere length $(\approx 2 \mu \mathrm{m})$ in insect flight muscle is comparable to that seen in vertebrate striated muscle, while sarcomere length $(\approx 9 \mu \mathrm{m})$ in insect visceral muscle is 34 times as long (Smith et al. 1966,Park and Shahabuddin 2000). Hence, sarcomeres were easily discernable by light microscopy in gut and ovary muscles. Myofilament and sarcomere structures were clearly visible in virus-laden gut muscles during both acute and persistent infection and we suggest that the fulminate arbovirus infectious process did not destroy gut muscles. Ultrastructural pathology was detected in both gut epithelial and gut muscle cells (Fig. 6). While this supports the findings of Bowers and colleagues (1995), this pathology did not appear widespread. Visceral muscles of the ovarian sheath did not support virus replication and remained refractory until at least day 18 p.i.

Because it was previously documented that permissive and refractory tissue co-existed in response to standard inoculum dose, we chose to challenge cell onset of virus detection and refractoriness with a high titer inoculum. Following high-titer virus challenge, virus antigen was detected earlier in the anterior midgut muscles, fat bodies, salivary glands, and thoracic musculature when compared with standard-virus titer inoculum (Bowers et al. 1995). This suggests that the anterior midgut muscles responded to an increased MOI while the muscles associated with the posterior midgut, hindgut or ovary did not respond to this increased inoculum. Possibly, virus receptors are more numerous on responsive muscles or that different 
cellular "tolerances" to virus reflects organ-specific functions. While all three examples of muscles (midgut, hindgut, and ovary) are classified as visceral, their physiology differs between different organs. Messer and Brown (1995) report quantitative and qualitative electrophysiological differences between mosquito hindgut and oviduct preparations. Hindguts exhibited irregular peristaltic contractions, while oviducts exhibited regular, sometimes synchronous contractions. Such differences reflect physiological demands associated with bloodfeeding, peristalsis, and oviposition respectively. Furthermore, functional diversity of insect visceral muscles may reflect differences in contractile protein isoforms as described for myosin heavy chain in Drosophila (Zhang and Berstein 2001). Myosin heavy chain is a molecular motor that binds actin and we suggest that organ-specific muscle associations may reflect muscle-specific physiology. Apparently, the fulminate infection of visceral muscles does not alter structure, and more research is needed to assess potential alterations in muscle related physiology.

Cell-to-cell junctions are essential for tissue organization. The BL is a dynamic connective tissue component that demarcates epithelial structures and influences developmental morphogenesis (Hay 1991). Cell-to-cell junctions between muscles were prominent, providing a scaffold for the insect gut and a netted sleeve for the insect ovary (Figs. 4 and 5). Muscle BL was thinner than the BL of the gut and ovary. Ultrastructural differences in cell-to-cell junctions and the BL were observed between the muscle: gut and muscle: ovary interface. Cell-to-cell junctions between gut muscles and gut epithelium could serve to create forces integral to withstanding the tremendous gut expansion following bloodfeeding. These physical "stays" imply that the bundles do not slide but provide a functional unit of cohesive strength. The midgut BL was detached, thrown up into folds, multilayered, possessed characteristic vertical hatch marks, all reflective of organ response to peristalsis. Wispy strands of muscle surrounded the ovaries and provided a cell-to-cell junction-free sleeve that would permit the sliding action necessary for growth and cyclic oviposition of egg batches. The ovary BL closely approximated the organ surface and was characterized by a confluent single layer with occasional rifts.

Basal lamina thickness differed between three strains of Ae. albopictus, but no significant correlations between BL thickness and dengue-1 virus dissemination was found between individuals (Thomas et al. 1993). Vertical transmission has been well documented for the flaviviruses and bunyaviruses (Leake 1984, Fontenille et al. 1997). While, evidence for transovariol transmission of the alphaviruses has been documented (Dhileepan et al. 1996, Fulhorst et al. 1994 and Mitchell et al. 1992), this transmission cycle does not appear to be a major contribution in nature (Leake 1984). Ross River virus and SIN were isolated from emerged field-caught larvae (males and females; Dhileepan et al. 1996) and low levels of vertical transmission of Ross River and Sindbis virus have been detected in the laboratory (Kay 1982). Eastern equine encephalitis virus was isolated from Ae. albopictus sampled from the wild in Florida (Mitchell et al. 1992). Fulhorst and colleagues (1994) report finding western equine encephalitis virus in Ae. dorsalis caught as larvae and these researchers document that their data "augments the limited experimental and field evidence for vertical transmission of the alphaviruses." We speculate that the thick BL surrounding the ovary (three times thicker than muscle BL) might in some manner hinder virus attachment, offering one possible explanation for the less frequent documentation and low level detection of vertical transmission of the alphaviruses.

Visceral muscles associated with different organs were probed with SIN revealing differential responses to this alphavirus. Presumably host cell factors could participate by interfering with virus replication in the midgut muscles or initiation of immune response such as an anti-virus response (clearance); uninhibited virus replication in the hindgut muscles such as incompetent immune response (persistence); the absence of receptors, block at entry or defective replication in the ovary muscles (refractoriness; Clements 1996). RNA interference (RNAi), an anti-virus 
defense mechanism (Campbell et al. 2008), or Toll signal transduction pathways (Sanders et al. 2005) might operate in the midgut muscle-specific cell clearance of virus antigens.

During Drosophila development, the midgut is derived from endoderm while the foregut and hindgut are derived from ectoderm (Martin and Bate 2001). The midgut endoderm requires the presence of circular visceral mesoderm, while the hindgut ectoderm can develop in the absence of visceral mesoderm, suggesting distinctly different morphogenetic events in the development of the midgut and the hindgut. Circular and longitudinal musculatures of the midgut originate from different regions of the mesodermal analage; both longitudinal visceral musculature of the midgut (Klapper 2000) and circular visceral musculature of the hindgut (Klapper et al. 2001) persist through metamorphosis into the adult in D. melanogaster. This absence of dedifferentiation and reorganization of larval tissue during metamorphosis might make it possible that persisting visceral cells have functions similar to those of the imaginal midgut epithelium during metamorphosis. Bernick and co-workers (2007) suggest that because overlapping bands are observed in Ae. aegypti larvae, it is quite possible that a common developmental element exists between the mosquito and drosophila development.

Selective muscle band degeneration was observed following the larval to pupal transition in Ae. aegypti (Bernick et al. 2008). Individual muscle cells underwent atrophy at varying times in the puparium, while cell death was not observed. These authors suggest that retention of the sarcolemma during myofilament degeneration could conceivably retain neuromuscular connectivity during development making retention of muscle integrity in the adult possible. These authors suggest that the metamorphosis of midgut visceral muscles may play a role in maintaining gut innervation in the pupa and stimulate nervous development in the adult. Muscle to adjacent muscle cell-to-cell junctions seen in our study (Figs. 4 and 5) would provide the means for structural retention of such a muscle scaffold during metamorphosis as well as potential retention of the sarcolemma studded with integral receptor molecules. The structurefunction relationships of insect visceral muscles reflect the organ-specific needs of the organism, and we speculate that varying developmental history, differences in the structural, physiological and biochemical milieu in the adult mosquito contribute to the differential phenotypic expression of SIN. We suggest that SIN virus can be used as a probe to detect cellular differences at the organismal level and further our understanding of the invertebrate host modulation of an alphavirus infection.

\section{Acknowledgments}

The research described was supported by Grant Number R15AI060654 from the National Institute of Allergy and Infectious Diseases; The Florida Mosquito Control Association T. Miller Wainwright Student Research Scholarship, 2003; The University of North Florida - UNF Student Summer Research Grant, 2003-2006 and UNF Faculty Scholarship Grant, summer 2004. We thank Judy Ochrietor for her critical reading of this manuscript and David Wilson (Center for Instruction and Research Technology-UNF) for his expertise in computer graphics. The content is solely the responsibility of the authors and does not necessarily represent the official views of the National Institute of Allergy and Infectious Diseases or the National Institutes of Health.

\section{References Cited}

Bernick EP, Moffett SB, Moffett DF. Organization, ultrastructure, and development of midgut visceral muscle in larval Aedes aegypti. Tissue and Cell 2007;39:277-292. [PubMed: 17675126]

Bernick EP, Moffett SB, Moffett DF. Ultrastructure and morphology of midgut visceral muscle in early pupal Aedes aegypti mosquitoes. Tissue and Cell 2008;40:127-141. [PubMed: 18160088]

Bowers DF, Abell BA, Brown DT. Replication and tissue tropism of the alphavirus Sindbis in the mosquito Aedes albopictus. Virology 1995;202:1-12. [PubMed: 7676618]

Bowers DF, Coleman CG, Brown DT. Sindbis virus-associated pathology in Aedes albopictus (Diptera: Culicidae). J Med Entomol 2003;40:698-705. [PubMed: 14596286] 
Brown, DT.; Condreay, LD. Replication of alphaviruses in mosquito cells. In: Schlesinger, S.; Schlesinger, MJ., editors. The Togaviridae and flaviridae. Academic; New York: 1986. p. 171-207.

Burge BW, Pfefferkorn ER. Isolation and characterization of conditional-lethal mutants of Sindbis virus. Virology 1966a;30:204-213. [PubMed: 5919228]

Burge BW, Pfefferkorn ER. Complementation between temperature sensitive mutants of Sindbis virus. Virology 1966b;30:214-223. [PubMed: 5919229]

Campbell CL, Keene KM, Brackney DE, Olson KE, Blair CD, Wilusz J, Foy BD. Aedes aegypti uses RNA interference in defense against Sindbis virus infection. BMC. Microbiology 2008;8:47-58. [PubMed: 18366655]

Chapman, RF. The insects: structure and function. Cambridge University Press; Cambridge: 1999.

Clements, AN. The biology of mosquitoes: structure of the adult alimentary canal. Vol. 2. CAB1 Publishing; Hall, New York: 1996.

Dhileepan K, Azuolas JK, Gibson CA. Evidence of vertical transmission of Ross River and Sindbis viruses (Togaviridae: Alphavirus) by mosquitoes (Diptera: Culicidae) in southeastern Australia. J Med Entomol 1996;33:180-182. [PubMed: 8906927]

Fontenille D, Diallo M, Mondo M, Ndiaye M, Thonnon J. First evidence of natural vertical transmission of yellow fever virus in Aedes aegypti, its epidemic vector. Trans Royal Soc Trop Med Hyg 1997;91:533-535.

Fulhorst CF, Hardy JL, Eldridge BF, Presser SB, Reeves WC. Natural vertical transmission of western equine encephalomyelitis virus in mosquitoes. Science 1994;263:676-678. [PubMed: 8303276]

Gaidamovich, AYa; Khutoretskaya, NV.; Lvova, AI.; Sveshnikova, NA. Immunofluorescent staining study of the salivary glands of mosquitoes infected with group A arboviruses. Intervirology 1973;1:193-200. [PubMed: 4590688]

Gighoh MEC. The female reproductive system of Anopheles gambiae melas II. The ovary Riv Malariol 1964;43:265-275.

Hay, ED. Cell biology of extracellular matrix. 2. Plenum; New York: 1991.

Higgs, S.; Beaty, BJ. The Biology of Disease Vectors. University Press of Colorado; Boulder, CO: 1996. Rearing and containment of mosquito vectors; p. 595-605.

Jackson AC, Bowen JC, Downe AER. Experimental infection of Aedes aegypti (Diptera: Culicidae) by the oral route with Sindbis virus. J Med Entomol 1993;30:332-337. [PubMed: 8384663]

Kay BH. Three modes of transmission of Ross River virus by Aedes vigilax (Skuse). Aust J Exp Med Sci 1982;60:339-344.

Klapper R. The longitudinal visceral musculature of Drosophila melanogaster persists through metamorphosis. Mech Develop 2000;95:47-54.

Klapper R, Heuser S, Strasser T, Janning W. A new approach reveals syncytia with the visceral musculature of Drosophila melanogaster. Development 2001;128:2517-2524. [PubMed: 11493568]

Kramer LD, Hardy JL, Presser SB, Houk EJ. Dissemination barriers for western equine encephalomyelitis virus in Culex tarsalis infected after ingestion of low viral doses. Am J Trop Med Hyg 1981;23:190_ 197. [PubMed: 7212166]

Leake, CJ. Transovarial transmission of arboviruses by mosquitoes. In: Mayo, CA.; Harrap, KA., editors. Vectors in Virus Biology. Academic Press; London, United Kingdom: 1984. p. 63-91.

Martin BS, Bate M. Hindgut visceral mesoderm requires an ectodermal template for normal development in Drosophila. Development 2001;18:233-242.

Messer AC, Brown MR. Non-linear dynamics of neurochemical modulation of mosquito oviduct and hindgut contractions. J Exper Biol 1995;198:2325-2336. [PubMed: 7490571]

Mitchell CJ, Niebylski ML, Smith GC, Karabatsos N, Martin D, Mutebi JP, Craig GB Jr, Mahler MJ. Isolation of eastern equine encephalitis virus from Aedes albopictus in Florida. Science 1992;257:526-527. [PubMed: 1321985]

Murphy, FA.; Whitfield, SG.; Sudia, WD.; Chamberlain, RW. Mechanisms of invertebrate vector parasite relations. In: Maramorosch, K.; Shope, RE., editors. Invertebrate immunity. Academic; New York: 1975. p. 25-48.

Park SS, Shahabuddin M. Structural organization of posterior midgut muscles in mosquitoes, Aedes aegypti and Anopheles gambiae. J Struct Biol 2000;129:30-37. [PubMed: 10675294] 
Raikova AP, Khutoretskaya NV, Tsilinsky Ya, Lvov DK, Klimenko SM. Morphogenesis of Sindbis virus in cells of the intestine and of the salivary glands of Aedes aegypti mosquitoes. Vopr Virusol 1973;18:283-289.

Renz D, Brown DT. Characteristics of Sindbis virus temperature-sensitive mutants in cultured BHK-21 and Aedes albopictus (mosquito) cells. J Virol 1976;19:775-781. [PubMed: 987251]

Rosen L, Gubler D. The use of mosquitoes to detect and propagate Dengue viruses. Am J Trop Med Hyg 1974;23:1153-1160. [PubMed: 4429185]

Sanders HR, Foy BD, Evans AM, Ross LS, Beaty BJ, Olson KE, Gill SS. Sindbis virus induces transport processes and alters expression of innate immunity pathway genes in the midgut of the disease vector, Aedes aegypti. Insect Biochem Mol Biol 2005;35:1293-1307. [PubMed: 16203210]

Seron TJ, Hill J, Linser PJ. A GPI-linked carbonic anhydrase expressed in the larval mosquito midgut. J Exper Biol 2004;207:4559-4572. [PubMed: 15579552]

Shope, RE. Alphavirus disease. In: Fields, BN., editor. Virology. Raven Press; New York: 1985. p. 931-953.

Smith DS, Gupta BL, Smith U. The organization and myofilament array of insect visceral muscles. J Cell Sci 1966;1:49-57. [PubMed: 5929807]

Thomas RE, Wu WK, Verleye D, Rai KS. Midgut basal lamina thickness and dengue-1 virus dissemination rates in laboratory strains of Aedes albopictus (Diptera: Culicidae). J Med Entomol 1993;30:326-331. [PubMed: 8459409]

Vo M, Bowers DF. Arbovirus lifecycle: links in a chain. Technical Bull FMCA 2005;7:31-34.

Zhang S, Bernstein SI. Spatially and temporally regulated expression of myosin heavy chain alternative exons during Drosophila embryogenesis. Mech Develop 2001;101:35-45. 
Actin Distribution

Phalloidin Labeled

Fig. 1. figure in color.)

\section{Anterior Midgut}

A

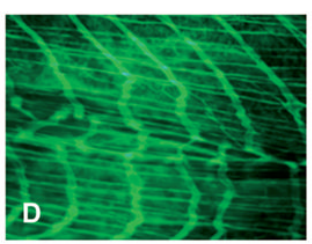

Posterior Midgut

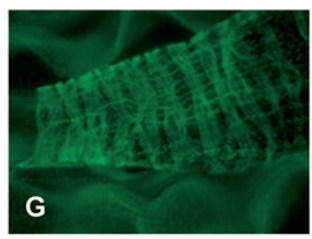

Hindgut

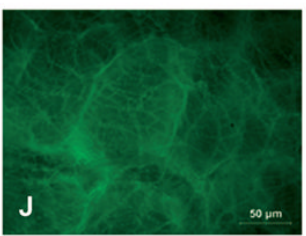

Ovary
Virus Antigen Distribution Antibody Labeled

Day 2-4 p.i.

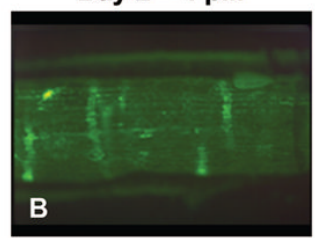

Day 10 p.i.
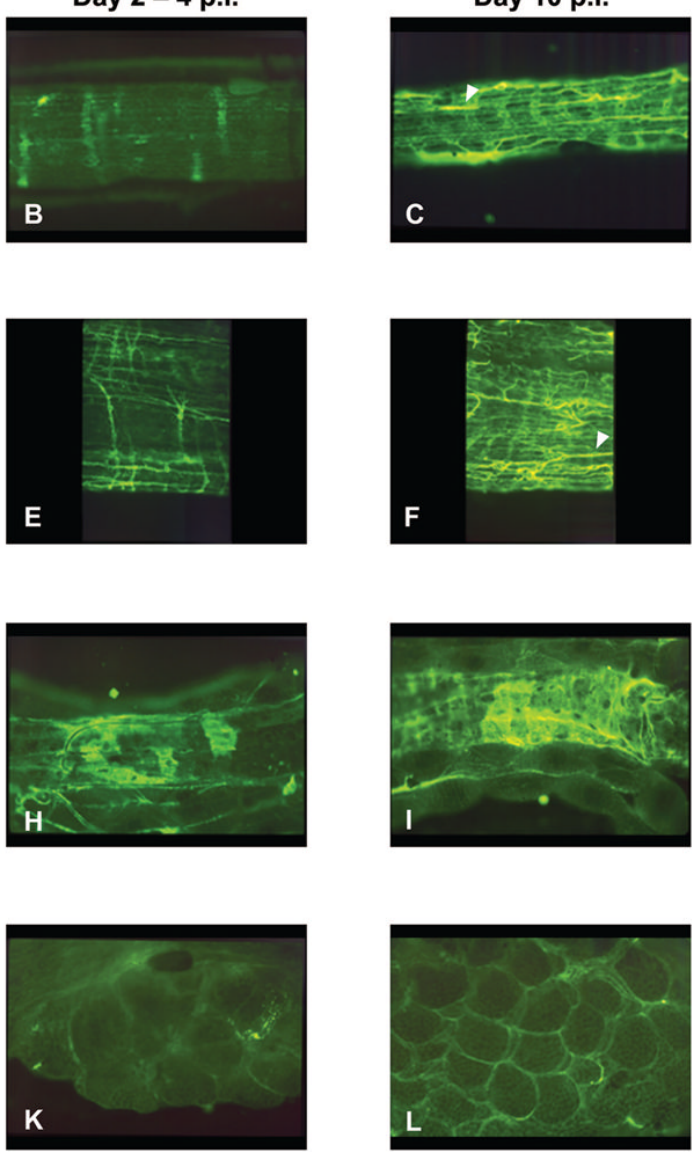

Comparison of actin distribution with the spatial pattern of virus infection at day 2-4 (acute infection) and day 10 p.i. (persistent infection) in whole-mount mosquito tissues. Virus antigen $(\mathrm{B}, \mathrm{E}$, and $\mathrm{H})$ mimicked actin distribution $(\mathrm{A}, \mathrm{D}$, and $\mathrm{G})$ during acute infection in gut-associated muscles. During persistent infection, virus antigen was cleared from anterior and posterior midgut muscles ( $\mathrm{C}$ and $\mathrm{F}$ ), become more noticeable in the tracheoles (arrowheads; $\mathrm{C}$ and $\mathrm{F}$ ), and persisted in hindgut-associated muscles ( $\mathrm{H}$ and $\mathrm{I}$ ). Note that the seemingly apparent brightness in $\mathrm{C}$ and $\mathrm{F}$ is because of the labeling of SIN in the trachea at day 10 p.i., not the muscle bands. Virus antigen (K and L) was not observed in ovary-associated muscles $(\mathrm{J})$ at anytime. Asterisk indicates cross-sharing of circular and longitudinal muscle bundles. (Online 
Posterior Midgut

\section{Hindgut}

Fig. 2.

\section{Mock}

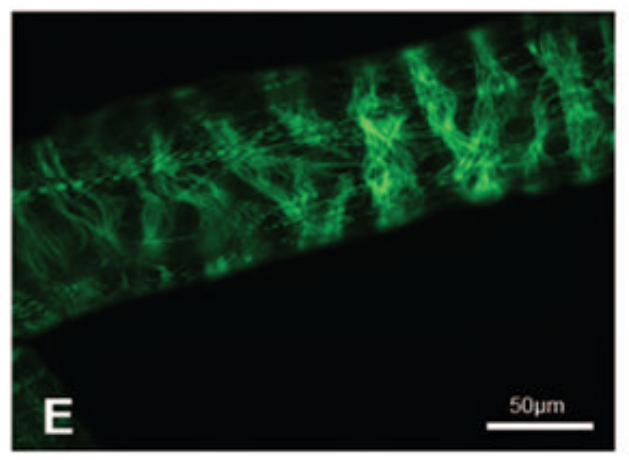

SIN Infected
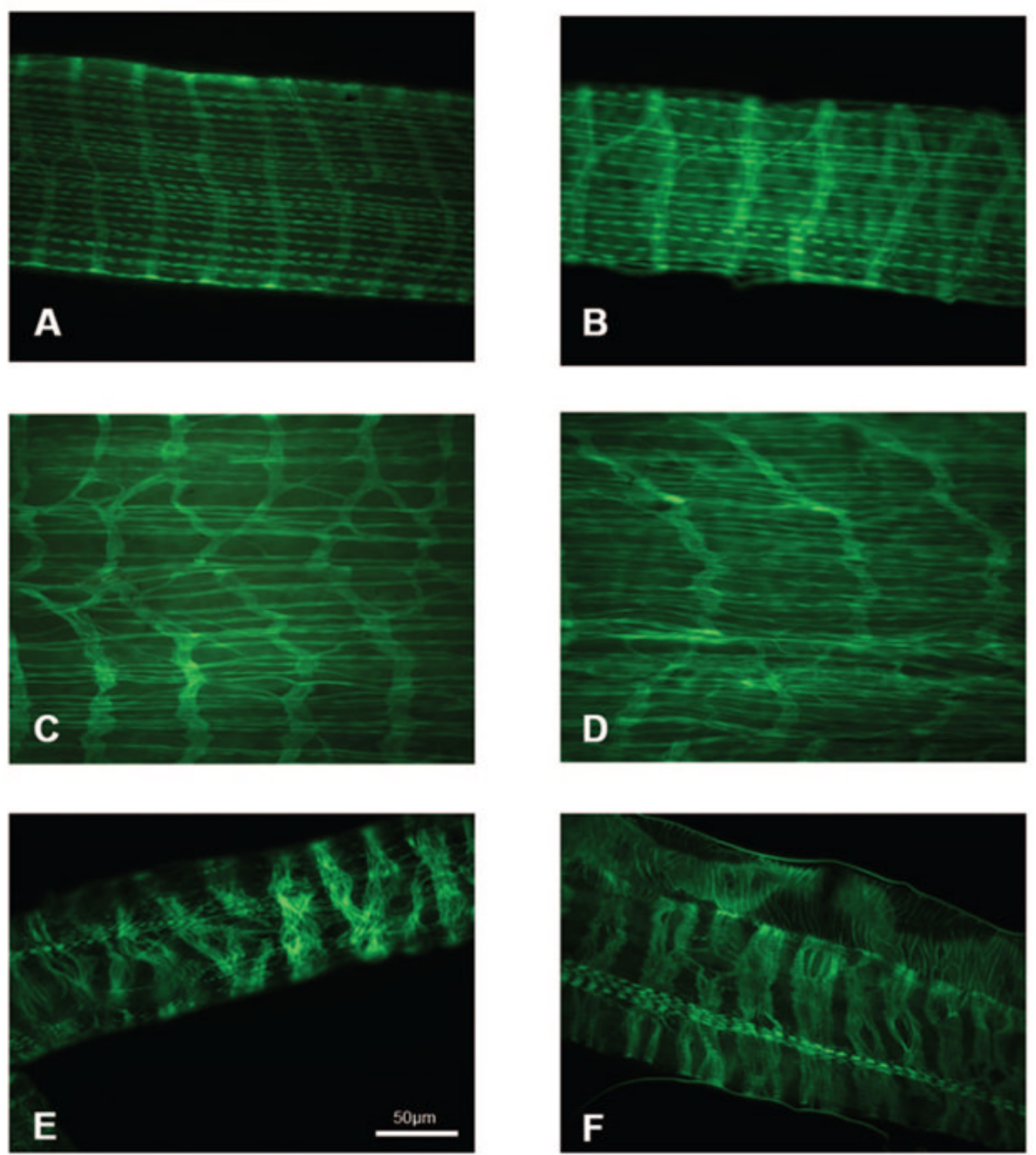

Temporal analysis of actin distribution in gut-associated muscles of Ae. albopictus following mock (uninfected) and SIN infection at $18 \mathrm{~d}$ p.i. Actin distribution in the anterior midgutassociated muscles reveal circular and longitudinal muscle bundles (A and D). Cross-sharing of muscle fibers are prominent in the circular muscle bundles and regular sarcomere distribution is observed most notably in the longitudinal muscles. Actin distribution in the posterior midgut-associated muscles likewise revealed circular and longitudinal muscle bundles ( $\mathrm{C}$ and $\mathrm{D})$. Cross-sharing of circular muscles was observed but sarcomere distribution is less apparent in this region of the gut. Actin distribution in the hindgut revealed a predominance of circular muscles and few strands of longitudinal muscle bundles. No overt difference in actin distribution was observed in any of these three gut regions at day 18 p.i. (Online figure in color.) 


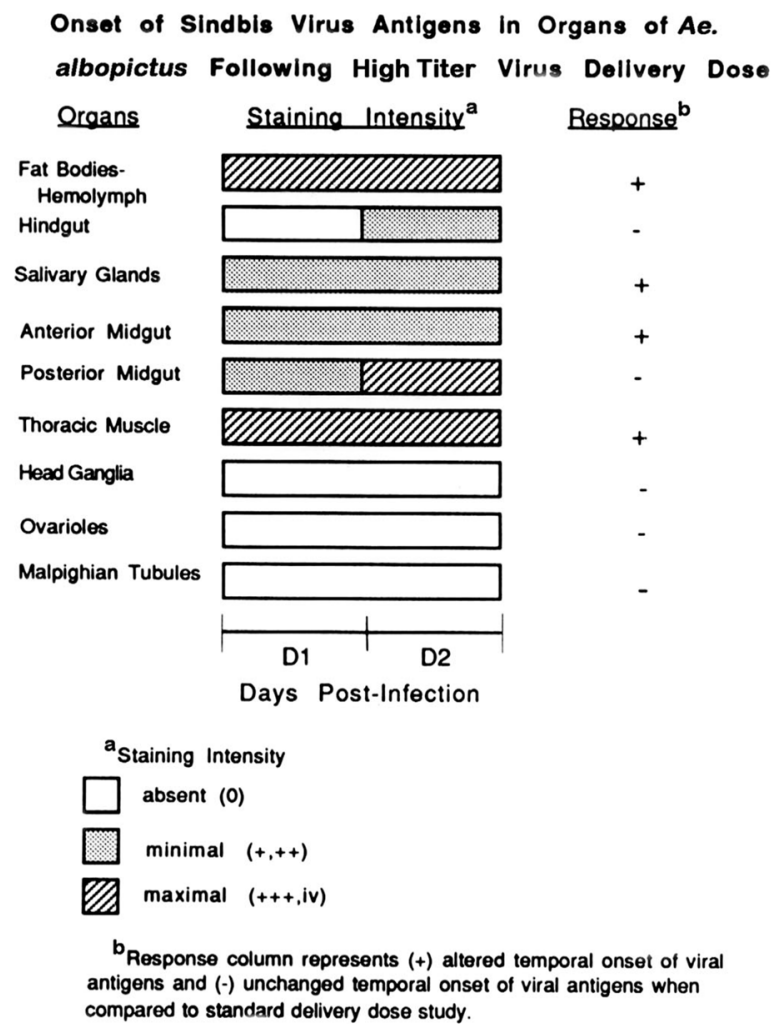

Fig. 3.

Onset of SIN antigens in organs of Ae. albopictus in response to increased inoculum titer. Insects were infected parenterally with 850,000 PFU SIN, organs were dissected at indicated days p.i. and acetone fixed to slides. Virus antigens were localized by indirect immunofluorescence staining using rabbit-produced SIN antiserum and FITC conjugatedsecondary antibody. Tissues were scored as $0,+,++,+++$, and iv (see Materials and Methods), and compared with standard infective inoculum (Bowers et al. 1995). 

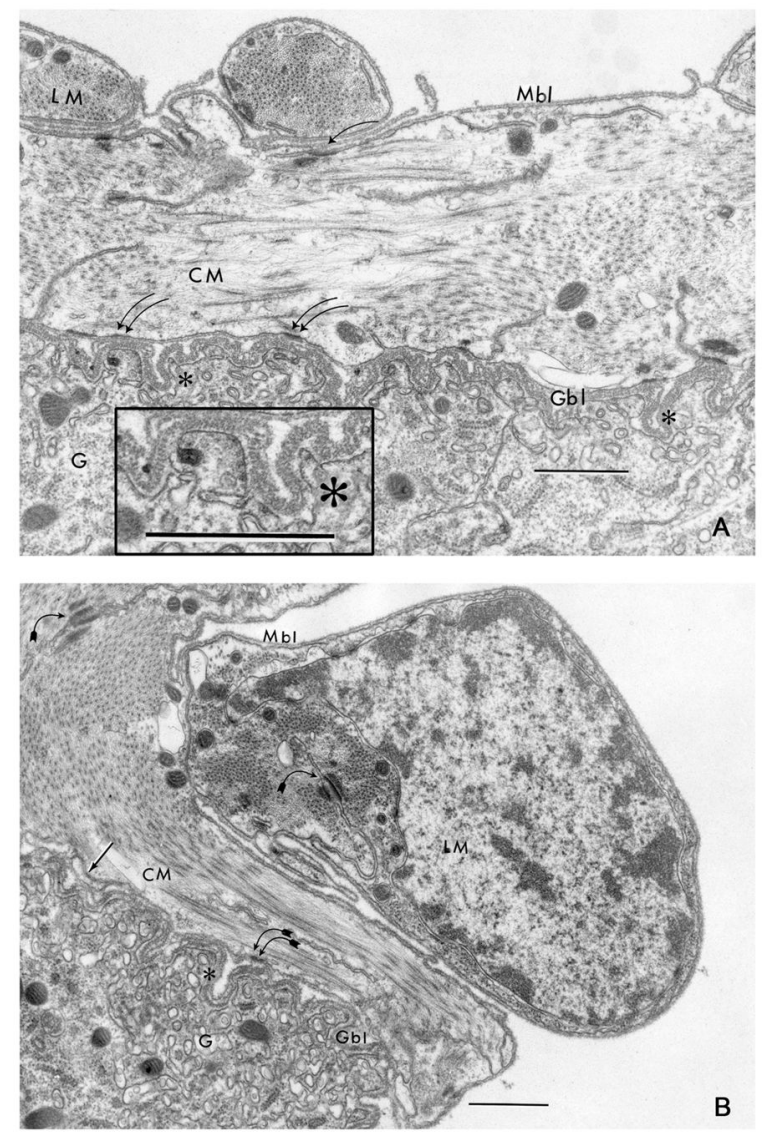

Fig. 4.

The circular muscle (CM) and longitudinal muscle (LM) interface between the gut $(\mathrm{G})$ outer surface is revealed by ultrastructural analysis (A and B). Muscle basal lamina (Mbl) is relatively thin and single-layered, while gut basal lamina $(\mathrm{Gbl})$ is relatively thick and multilayered (arrow) revealing characteristic vertical crosshatch marks (asterisk; inset). Muscle cells present a smooth contour, while the basal aspect of the enterocytes is highly convoluted and deeply folded (asterisk). The Mbl and Gbl are closely juxtaposed, but this approximation is not confluent down into folds (asterisk). Cell junctions between muscle cells (curved arrow) and between muscle cells and enterocytes (double curved arrows) are noted. Scale bar $=0.5 \mu \mathrm{m}$. 

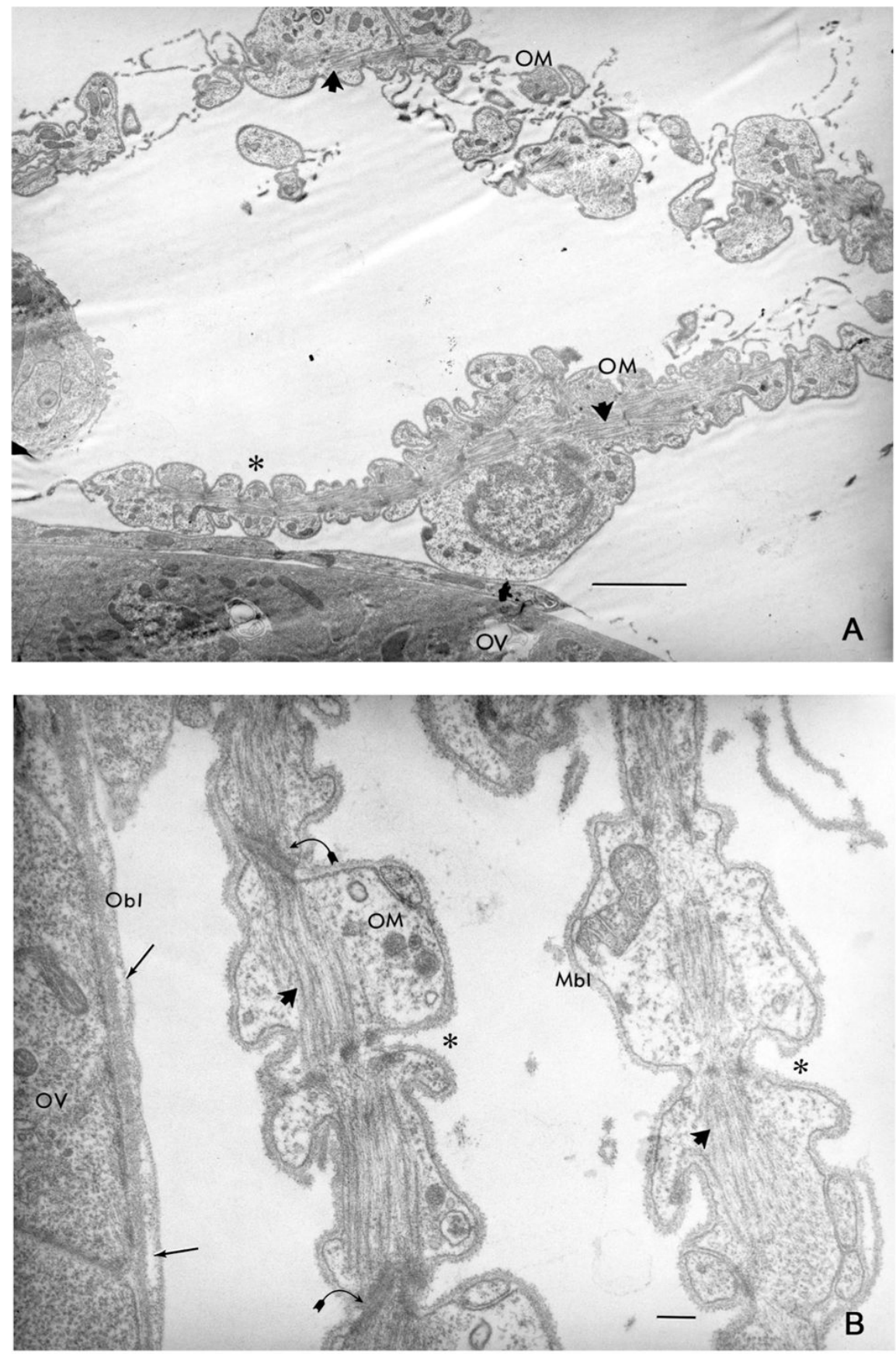

Fig. 5.

Ultrastructural analysis of muscle: ovary interface reveals the approximation of the visceral muscle basal lamina $(\mathrm{Mbl})$ and ovary basal lamina $(\mathrm{Obl})$ interaction (A and B). Ovary visceral muscles $(\mathrm{OM})$ in single or double layers loosely surround the ovary $(\mathrm{OV})$. Bundles of myofilaments in longitudinal section (arrowheads) are centrally located in visceral muscles, cells that are morphologically scalloped (asterisk) in an accordion-style. The Mbl are relatively thin and single layered, while the $\mathrm{Obl}$ are relatively thick with prominent rifts (arrows). Cell junctions are noted between muscle cells (curved arrows) but were not observed between muscles and the ovary surface. Scale bar $=$ (A) $0.1 \mu \mathrm{m}$, (B) $0.5 \mu \mathrm{m}$. 


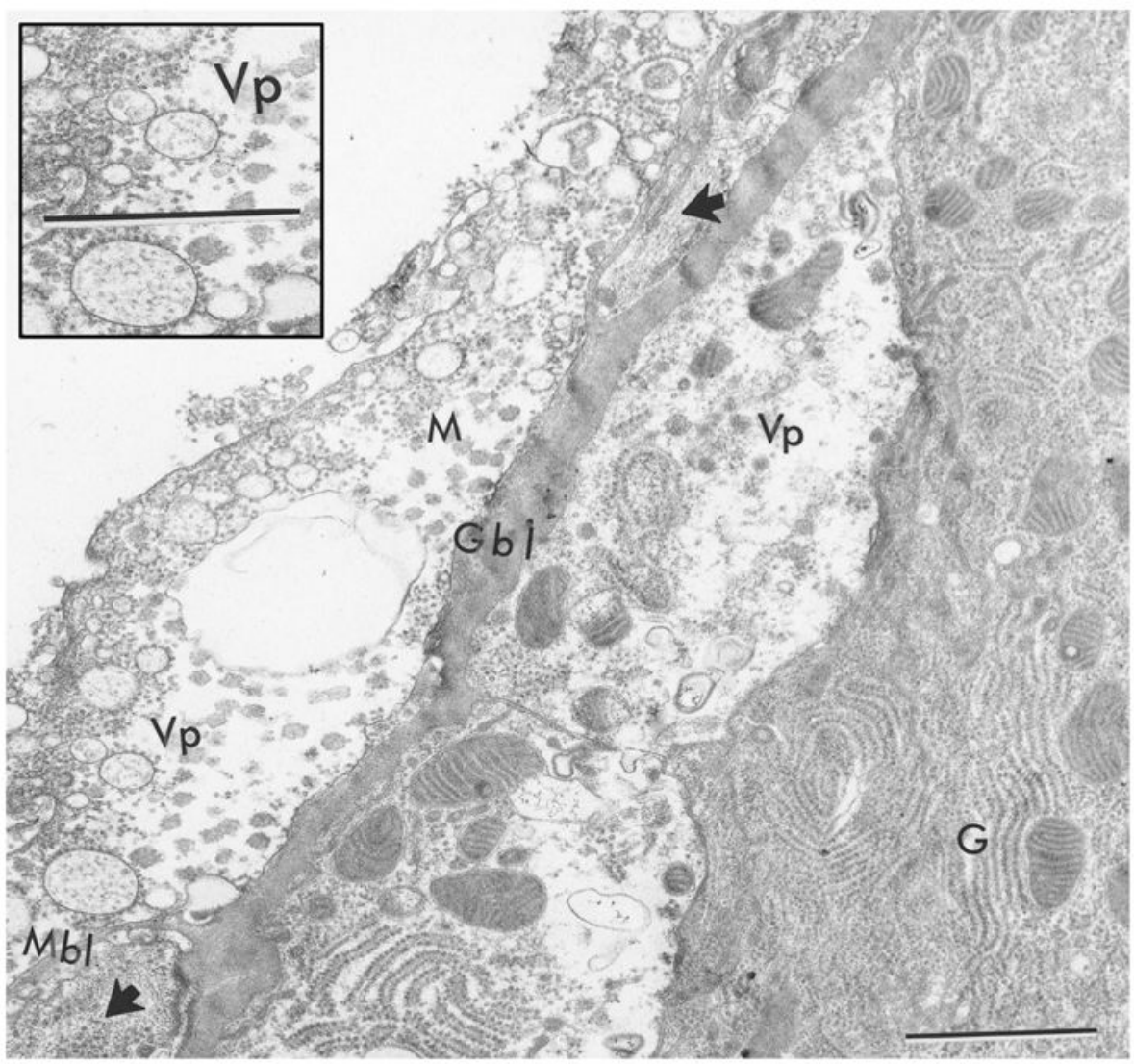

Fig. 6.

Ultrastructural evidence of virus-associated pathology in midgut cell and midgut-associated muscle cell at $5 \mathrm{~d}$ p.i. with SIN. Cytopathology was observed in gut cells $(\mathrm{G})$ above the basal lamina $(\mathrm{Gbl})$ and in visceral muscle cells $(\mathrm{M})$ beneath the basal lamina (Mbl). Note vacuolated spaces in cells and virus particles, both indicative of virus pathology $(\mathrm{Vp})$. Inset shows enlargement of $\mathrm{Vp}$. Arrowheads denote myofilaments in cross-section and longitudinal-section in visceral muscles. Scale bar $=0.5 \mu \mathrm{m}$. 